\title{
Clinical Study \\ Fungal Exposure and Low Levels of IL-10 in Patients with Sarcoidosis
}

\author{
Marjeta Terčelj, ${ }^{1}$ Sanja Stopinšek, ${ }^{2}$ Alojz Ihan, ${ }^{2}$ Barbara Salobir, \\ Saša Simčič, ${ }^{2}$ and Ragnar Rylander ${ }^{3}$ \\ ${ }^{1}$ Department of Respiratory and Allergic Diseases, The University Medical Centre, Ljubljana, Slovenia \\ ${ }^{2}$ Institute of Microbiology and Immunology, Faculty of Medicine, University of Ljubljana, 1000 Ljubljana, Slovenia \\ ${ }^{3}$ Biofact Environmental Health Research Center, Björkåsvägen 21, 44391 Lerum, Sweden
}

Correspondence should be addressed to Ragnar Rylander; envhealth@biofact.se

Received 9 May 2014; Accepted 9 July 2014; Published 7 August 2014

Academic Editor: Leif Bjermer

Copyright (C) 2014 Marjeta Terčelj et al. This is an open access article distributed under the Creative Commons Attribution License, which permits unrestricted use, distribution, and reproduction in any medium, provided the original work is properly cited.

Background and Objectives. Sarcoidosis is an inflammatory disease with increased levels of inflammatory cytokines. Previous studies have shown a relation between the degree of granuloma infiltration and serum cytokine levels, except for interleukin- (IL-) 10. The aim of the study was to further investigate the serum levels of IL-10 in patients with sarcoidosis and relate them to fungal exposure in terms of the amount of fungi in the air of their homes and $\beta$-glucan in bronchoalveolar lavage (BAL) fluid. Methods. Patients with sarcoidosis $(n=71)$ and healthy controls $(n=27)$ were enrolled. IL-10 was determined in serum. BAL was performed and the amount of $\beta$-glucan was measured. Domestic exposure to fungi was determined by measuring airborne $\beta$ - $\mathrm{N}$-acetylhexosaminidase (NAHA) in the bedrooms. Results. At high levels of fungal exposure (domestic fungal exposure and $\beta$-glucan in BAL), serum IL-10 values were lower than at low and intermediate exposure levels. Conclusion. The low serum IL-10 values at high fungal exposure suggest that fungal cell wall agents play a role in granuloma formation in sarcoidosis by inhibiting the secretion of the anti-inflammatory cytokine IL-10.

\section{Introduction}

Sarcoidosis is an inflammatory disease, often leading to granuloma formation $[1,2]$. Several studies demonstrate that the amounts of inflammatory cytokines, particularly interleukin(IL-) 10 and IL-12, are elevated in serum and in bronchoalveolar lavage fluid [3-6]. Previous studies have demonstrated that exposure to fungi is a risk factor for sarcoidosis [7-9]. One fungal cell wall agent (FCWA) - $\beta$-glucan - can induce different changes in the immune system and granulomas, depending on dose and means of administration (review in [10]). The formation of granuloma can be suppressed by IL10 [11]. Chitin is another FCWA that can induce immune changes, depending on the size of the particles [12].

In in vitro studies on the reactivity of peripheral blood mononuclear cells (PBMC), particulate $\beta$-glucan was found to induce the secretion of TNF $\alpha$, IL-6, IL-10, and IL-12 from
PBMC [13] with a higher secretion from PBMC taken from patients with sarcoidosis [14].

A clinical study evaluated the relation between the extent of granuloma infiltration using an $\mathrm{x}$-ray score and the amount of serum TNF $\alpha$, IL-6, IL-10, IL-12, angiotensin converting enzyme (ACE), and chitotriosidase (CTO) [15]. There was a linear relationship for all inflammatory mediators and markers except for IL-10. For this cytokine, there was an initial increase with an increased X-ray score but the values were lower at the highest X-ray scores. This suggests that a blocking of the normal secretion of IL-10, due to inflammation, might be a mechanism related to the risk of granuloma formation in sarcoidosis. This observation prompted the present study which comprises an evaluation of serum levels of IL-10 in patients with sarcoidosis in relation to fungal exposure in terms of exposure to fungi at their home and the amount of $\beta$-glucan in BAL. 


\section{Material and Methods}

2.1. Subjects. The subjects were recruited from the Department for Respiratory and Allergic Diseases at the University Medical Centre, Ljubljana, Slovenia, from July 2007 to October 2013. The department is one of the national centres for patients with sarcoidosis. For the diagnosis the ERS/ATS criteria [16] are used. The routine at the clinic is to make bronchoscopy with 5 to 10 transbronchial biopsies of lung parenchyma and needle aspiration of mediastinal lymph nodes. Bronchoalveolar lavage (BAL) is made and the $\mathrm{CD} 4+/ \mathrm{CD} 8+$ ratio is determined. The presence of noncaseating granulomas is verified histologically. If a biopsy is not considered representative, the patient undergoes surgical pulmonary or lymph node biopsy. Aspiration is performed from the right, upper lobe for culturing pathogenic fungi and bacteria including tuberculosis. Most biopsies are stained (silver staining, Gomori) to identify the presence of fungal infection. IgA, IgM, and IgG antibodies against Candida spp. and Aspergillus spp. are determined as well as mannan antigen in blood. Diagnostic BAL and sputum on subjects with sarcoidosis demonstrated an absence of pathogenic fungi and TB.

This study comprises newly diagnosed cases of pulmonary sarcoidosis $(n=71)$ and healthy subjects $(n=26)$ were examined. All subjects were nonsmokers. The study was approved by the Ethical Committee at the University Medical Centre, Ljubljana (198/05/04), and written, informed consent was obtained.

2.2. Clinical Assessments. IL-10 was determined in serum using a commercial ELISA kit (Milenia Biotec, Germany, and Thermo Scientific, USA) and expressed as $\mathrm{mmol} / \mathrm{mL}$. Chitotriosidase (CTO) activity in serum was determined using $22 \mu \mathrm{M}$ 4-methylumbelliferyl- $\beta$-D-N,N ${ }^{\prime}, \mathrm{N}^{\prime \prime}$-triacetylchitotriosiose (Sigma) in citrate phosphate buffer ( $\mathrm{pH} 5.2$ ) and expressed as $\mathrm{nmol} / \mathrm{h} / \mathrm{mL}[17,18]$.

2.3. Analysis of $\beta$-Glucan. Samples of the BAL fluid were mixed with $20 \mathrm{~mL}$ of a solution containing $0.15 \mathrm{~mol} / \mathrm{L} \mathrm{KOH}$, $0.3 \mathrm{~mol} / \mathrm{L} \mathrm{KCl}$, and $0.1 \%$ polybrene and incubated at $37^{\circ} \mathrm{C}$ for 10 minutes. For the analysis of $\beta$-glucan, a commercially available method based on the reactivity of a Limulus extract was used. The BAL preparation was diluted in a protein blocking buffer (Biodispersing agent, Charles River, Charleston, SC, USA), kept in boiling water for two minutes, and further diluted in endotoxin free water (LAL, Charles River). Thereafter, $25 \mu \mathrm{L}$ was added to each of the four wells in a plate preprepared with a Limulus reagent specific for $\beta$-glucan and read in an automatic analyser (Endosafe PTS, Charles River). The lower limit for detection is $1 \mathrm{pg} / \mathrm{mL}$. Samples yielding a readout value of $<100 \mathrm{pg} / \mathrm{mL}$ were given the uniform value of 90 to save on reagents. For ethical reasons BAL was only performed in nine control subjects.

2.4. Fungal Exposure at Home. The exposure to fungi in the homes was determined by analysing the amount of airborne $\beta$-N-acetylhexosaminidase (NAHA) as a marker of fungal
TABLE 1: Basic characteristics of subjects with sarcoidosis. Mean and (SEM).

\begin{tabular}{lccl}
\hline Parameter & Controls & Sarcoidosis & \\
\hline$n$ & 26 & 71 & \\
Age years & $57.6(5.2)$ & $47.4(1.4)$ & \\
Females \% & 63 & 49 & \\
NAHA U/m & $19.0(5.0)$ & $34.1(3.9)$ & $P<0.001$ \\
B-glucan pg/mL & $122.5(16.1)$ & $474.5(38.6)$ & $P<0.001$ \\
CD4/CD8 ratio & & $7.5(0.7)$ & \\
CTO nmol/h/mL & & $775(68)$ & \\
sACE $\mu$ Kat $/ \mathrm{L}$ & & $0.49(0.03)$ & \\
\hline
\end{tabular}

TABLE 2: IL-10 values (pg/mL) in serum of subjects with sarcoidosis and controls. Mean, SEM and range.

\begin{tabular}{lcccccc}
\hline Subjects & $n$ & Mean & SEM & Minimum & Maximum & $P$ \\
\hline Controls & 26 & 10.3 & 2.8 & 1 & 63 & \\
Sarcoidosis & 71 & 16.6 & 3.7 & 1 & 270 & NS \\
\hline
\end{tabular}

cell biomass [19, 20]. Air samples (around 2000 L) were taken in the subject's bedroom using a filter and a fluorogenic enzyme substrate (4-methylumbelliferyl N-acetyl- $\beta$-Dglucosaminide, Mycometer A/S, Copenhagen, Denmark) was added to the filter. After an incubation period of around 30 minutes, set by room temperature, a developer was added, and the fluorescence of the liquid was read in a fluorometer (Picofluor, Turner Designs, Sunnyvale, CA, USA). The units read were divided by 10 to diminish methodological scatter and expressed as NAHA Units $/ \mathrm{m}^{3}$.

2.5. Statistical Analysis. Values in the different groups were calculated using SPSS W7 and were expressed as mean and standard error of the mean (SEM). Differences between groups were evaluated using the $t$-test or Fisher's exact test. A $P$ value of $\leq 0.05$ was considered statistically significant.

\section{Results}

3.1. Characteristics of Test Subjects. Basic characteristics of the subjects are shown in Table 1.

The clinical values are typical for newly diagnosed sarcoidosis. There were four cases of skin sarcoidosis.

Serum IL-10 values are reported in Table 2.

There were no significant differences in mean values between controls and sarcoidosis. The maximum value was higher among subjects with sarcoidosis. In controls all $\beta$ glucan values were less than $90 \mathrm{pg} / \mathrm{mL}$ and NAHA values were less than $30 \mathrm{U} / \mathrm{m}^{3}$ except for one outlier (76).

Figure 1 illustrates serum levels of IL-10 among subjects with sarcoidosis in relation to $\beta$-glucan in BAL.

At higher amounts of $\beta$-glucan in BAL and NAHA in the homes, reflecting a higher exposure to fungi, there were no high levels of IL-10. At a break-off point of $750 \mathrm{pg} / \mathrm{mL}$ $\beta$-glucan, the mean IL-10 value in the lower $\beta$-glucan value group was $23.5 / 5.7$ as compared to 3.8/0.5 for the higher value group ( $P=0.026$, Mann-Whitney's test). At a break-off point 


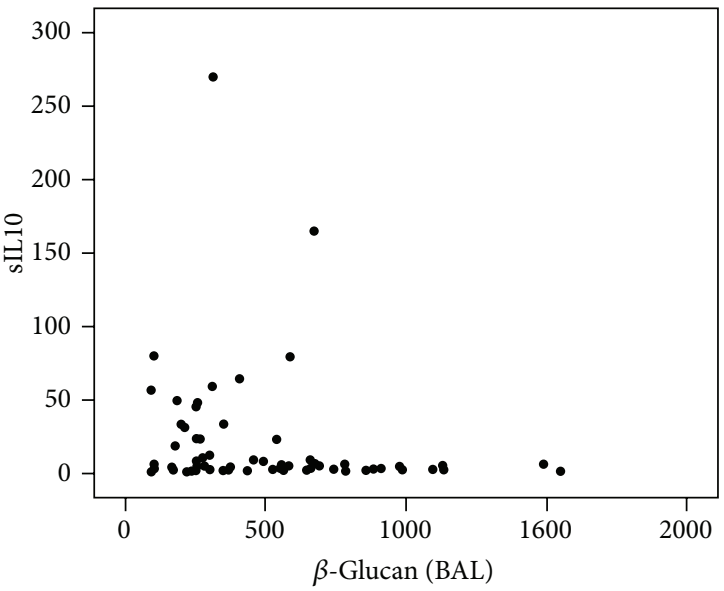

(a)

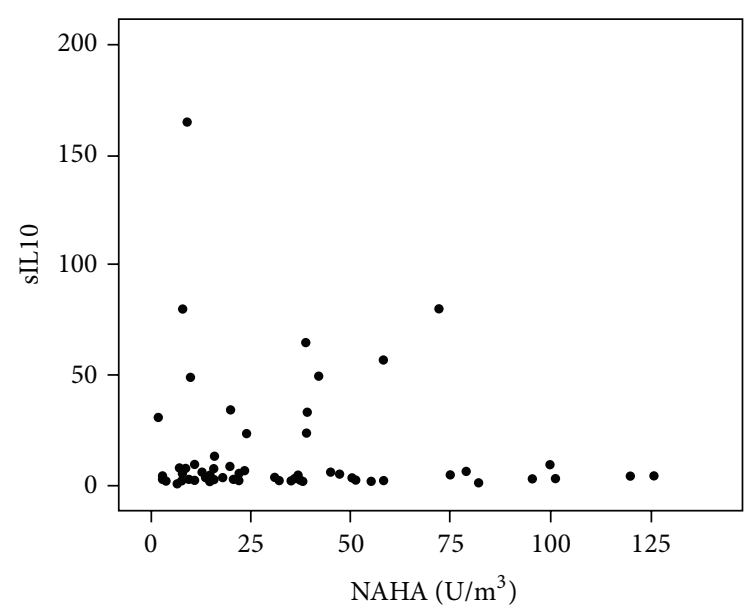

(b)

Figure 1: (a) Serum IL-10 in relation to the amount of $\beta$-glucan (BAL) pg/mL ( $n=70)$ and (b) NAHA in homes $(n=60)$ of subjects with sarcoidosis.

of NAHA of 75, the corresponding IL-10 values were 20.6/8.9, $n=65$, and $5.6 / 1.5, n=7$ ( $P=0.0001$, Fisher's exact test $)$.

\section{Discussion}

The major findings from the study are that the serum values of IL-10 were low at higher exposure to fungi, as illustrated by the domestic exposure and the amount $\beta$-glucan in BAL.

There are some limitations to the study. The number of subjects is fairly small, particularly in the high fungal exposure group. Measurements of NAHA were made in the homes but significant exposures could also have been present at the workplace. BAL for $\beta$-glucan determination could not be performed in all subjects because of ethical reasons.

A previous study has shown that values of IL-10 were lower at higher levels of CTO, a marker of disease severity [15]. In this study a similar decrease was present in relation to the fungal exposure at home. This suggests that there is a depression of IL-10 secretory capacity at higher exposure levels. Almost all cells of both the innate and adaptive arms of the immune system can express IL-10 including dendritic cells, macrophages, mast cells, natural killer cells, eosinophils, neutrophils, and T regulatory cells [21, 22]. This cytokine is one of the most important anti-inflammatory and immune suppressive cytokines. It affects the vascular system through inhibition of leukocyte-endothelial cell interaction and inhibition of proinflammatory cytokine and chemokine production by macrophages and lymphocytes [23]. In an animal model low levels of IL-10 were a predisposing factor for chronic fibrosis [24]. Of particular interest in sarcoidosis is that IL-10 can inhibit an experimental granulomatous inflammation [11] and that corticosteroids, which are usually used to treat the disease, have the capacity to enhance IL-10 production [25].

This opens up a new possible mechanism to explain the relation between fungal exposure and sarcoidosis [9]. It is tempting to speculate that the development of granulomas in sarcoidosis is initiated by an inflammation induced by inhaled $\beta$-glucan and that this inflammation at higher exposure levels depresses an important defence mechanism in terms of IL-10. Further work is required to assess this hypothesis. Although the focus in this context has been on fungi, it cannot be excluded that other microbial agents may induce similar reactions in terms of depression of the cellular capacity to secrete IL-10. To assess the importance of such agents, for example, Mycobacteria, studies on exposure levels as well as the effect on cellular mechanisms are required.

\section{Conclusions}

At a high environmental exposure to fungi, the level of serum IL-10 among subjects with sarcoidosis was low. This suggests that $\beta$-glucan could play a role in the granuloma formation in sarcoidosis by blocking a defence system in terms of IL-10 secretion. At high levels of fungal exposure patients with sarcoidosis have a depressed secretion of the antiinflammatory cytokine IL-10. This suggests a new mechanism for the development of granulomas in sarcoidosis.

\section{Conflict of Interests}

The authors declare that there is no conflict of interests regarding the publication of this paper.

\section{Authors' Contribution}

M. Terčelj and Barbara Salobir were responsible for the clinical work, S. Stopinšek, A. Ihan, and S. Simčič were responsible for the PBMC work, and R. Rylander was responsible for the environmental and glucan measures and the analyses of the data. All authors agree on the content of the paper. 


\section{Acknowledgment}

This paper received internal hospital funding only.

\section{References}

[1] M. C. Iannuzi, B. A. Rybicki, and A. S. Tierstein, "Sarcoidosis," The New England Journal of Medicine, vol. 357, pp. 2153-2165, 2007.

[2] R. P. Baughman, D. A. Culver, and M. A. Judson, "A concise review of pulmonary sarcoidosis," The American Journal of Respiratory and Critical Care Medicine, vol. 183, no. 5, pp. 573581, 2011.

[3] S. Nureki, E. Miyazaki, M. Ando et al., "Circulating levels of both Th1 and Th2 chemokines are elevated in patients with sarcoidosis," Respiratory Medicine, vol. 102, no. 2, pp. 239-247, 2008.

[4] R. M. Mroz, M. Korniluk, A. Stasiak-Barmuta, and E. Chyczewska, "Increased levels of interleukin-12 and interleukin18 in bronchoalveolar lavage fluid of patients with pulmonary sarcoidosis," Journal of Physiology and Pharmacology, vol. 59, supplement 6, pp. 507-513, 2008.

[5] M. Hata, K. Sugisaki, E. Miyazaki, T. Kumamoto, and T. Tsuda, "Circulating IL-12 p40 is increased in the patients with sarcoidosis, correlation with clinical markers," Internal Medicine, vol. 46, no. 17, pp. 1387-1394, 2007.

[6] U. Oltmanns, B. Schmidt, S. Hoernig, C. Witt, and M. John, "Increased spontaneous interleukin-10 release from alveolar macrophages in active pulmonary sarcoidosis," Experimental Lung Research, vol. 29, no. 5, pp. 315-328, 2003.

[7] L. S. Newman, C. S. Rose, E. A. Bresnitz et al., "A casecontrol etiological study of sarcoidosis-environmental and occupational risk factors," American Journal of Respiratory and Critical Care Medicine, vol. 170, no. 12, pp. 1324-1330, 2004.

[8] A. S. Laney, L. A. Cragin, L. Z. Blevins et al., "Sarcoidosis, asthma, and asthma-like symptoms among occupants of a historically water-damaged office building," Indoor Air, vol. 19, no. 1, pp. 83-90, 2009.

[9] M. Terčelj, B. Salobir, and R. Rylander, "Airborne enzyme in homes of patients with sarcoidosis," Environmental Health, vol. 10, pp. 8-13, 2011.

[10] R. Rylander, "Organic dust induced pulmonary disease - The role of mould derived B-Glucan," Annals of Agricultural and Environmental Medicine, vol. 17, no. 1, pp. 9-13, 2010.

[11] H. H. Herfarth, S. P. Mohanty, H. C. Rath, S. Tonkonogy, and R. B. Sartor, "Interleukin 10 suppresses experimental chronic, granulomatous inflammation induced by bacterial cell wall polymers," Gut, vol. 39, no. 6, pp. 836-845, 1996.

[12] R. A. A. Muzzarelli, "Chitins and chitosans as immunoadjuvants and non-allergenic drug carriers," Marine Drugs, vol. 8, no. 2, pp. 292-312, 2010.

[13] S. Stopinšek, A. Ihan, B. Wraber et al., "Fungal cell wall agents suppress the innate inflammatory cytokine responses of human peripheral blood mononuclear cells challenged with lipopolysaccharide in vitro," International Immunopharmacology, vol. 11, no. 8, pp. 939-947, 2011.

[14] M. Terčelj, S. Stopinšek, A. Ihan et al., "In vitro and in vivo reactivity to fungal cell wall agents in sarcoidosis," Clinical and Experimental Immunology, vol. 166, no. 1, pp. 87-93, 2011.

[15] M. Terčelj, B. Salobir, M. Zupancic, B. Wraber, and R. Rylander, "Inflammatory markers and pulmonary granuloma infiltration in sarcoidosis," Respirology, vol. 19, pp. 225-230, 2014.
[16] "Association of sarcoidosis and other granulomatous disorders (WASOG) adopted by the ATS board of directors and by the ERS executive committee," The American Journal of Respiratory and Critical Care Medicin, vol. 160, pp. 736-755, 1999.

[17] E. Bargagli, D. Bennett, C. Maggiorelli et al., "Human chitotriosidase: a sensitive biomarker of sarcoidosis," Journal of Clinical Immunology, vol. 33, no. 1, pp. 264-270, 2013.

[18] M. Terčelj, B. Salobir, S. Simcic, B. Wraber, M. Zupancic, and R. Rylander, "Chitotriosidase activity in sarcoidosis and some other pulmonary diseases," Scandinavian Journal of Clinical and Laboratory Investigation, vol. 69, no. 5, pp. 575-578, 2009.

[19] A. M. Madsen, "NAGase activity in airborne biomass dust and relationship between NAGase concentrations and fungal spores," Aerobiologia, vol. 19, no. 2, pp. 97-105, 2003.

[20] R. Rylander, M. Reeslev, and T. Hulander, "Airborne enzyme measurements to detect indoor mould exposure," Journal of Environmental Monitoring, vol. 12, no. 11, pp. 2161-2164, 2010.

[21] R. Sabat, "IL-10 family of cytokines," Cytokine and Growth Factor Reviews, vol. 21, no. 5, pp. 315-324, 2010.

[22] T. H. S. Ng, G. J. Britton, E. V. Hill, J. Verhagen, B. R. Burton, and D. C. Wraith, "Regulation of adaptive immunity; the role of interleukin-10," Frontiers in Immunology, vol. 4, pp. 1-13, 2013.

[23] D. A. Smith, S. D. Irving, J. Sheldon, D. Cole, and J. C. Kaski, "Serum levels of the antiinflammatory cytokine interleukin-10 are decreased in patients with unstable angina," Circulation, vol. 104, no. 7, pp. 746-749, 2001.

[24] L. J. Zhang, W. D. Zheng, Y. X. Chen et al., "Antifibrotic effects of interleukin-10 on experimental hepatic fibrosis," HepatoGastroenterology, vol. 54, no. 79, pp. 2092-2098, 2007.

[25] D. F. Richards, M. Fernandez, J. Caulfield, and C. M. Hawrylowicz, "Glucocorticoids drive human $\mathrm{CD}^{+} \mathrm{T}$ cell differentiation towards a phenotype with high IL-10 and reduced IL-4, IL-5, and IL-13 production," European Journal of Immunology, vol. 30, no. 8, pp. 2344-2354, 2000. 


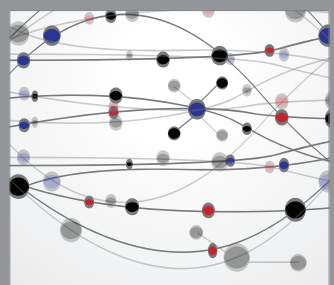

The Scientific World Journal
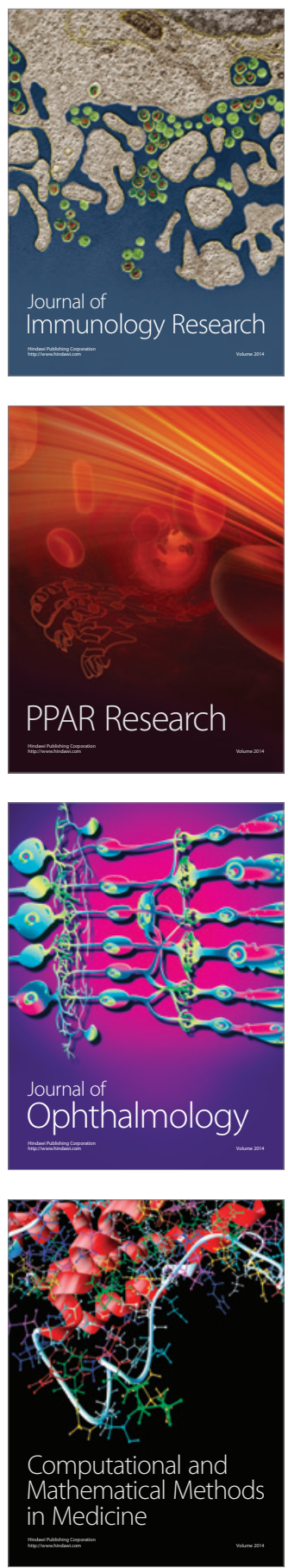

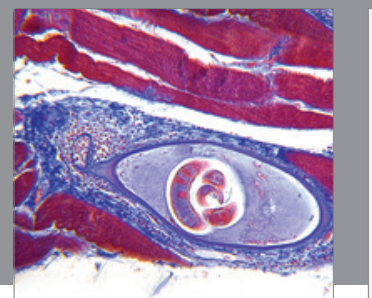

Gastroenterology

Research and Practice
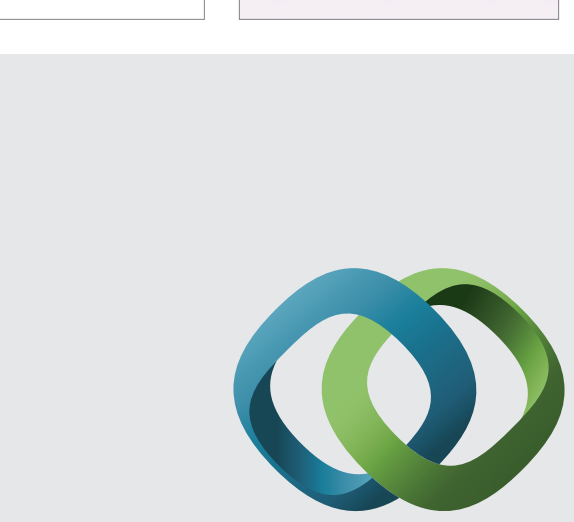

\section{Hindawi}

Submit your manuscripts at

http://www.hindawi.com
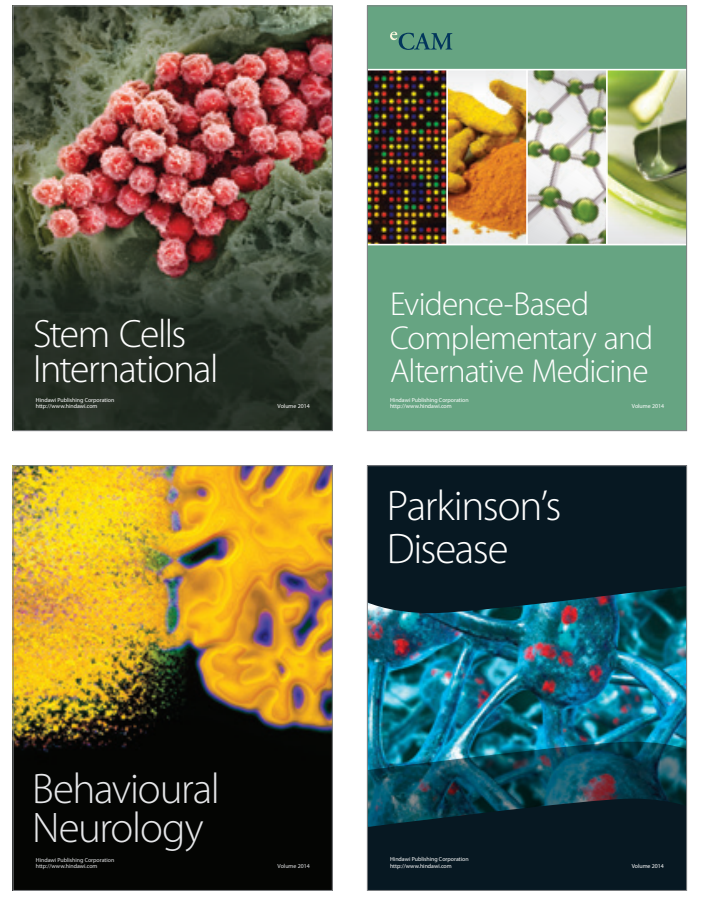
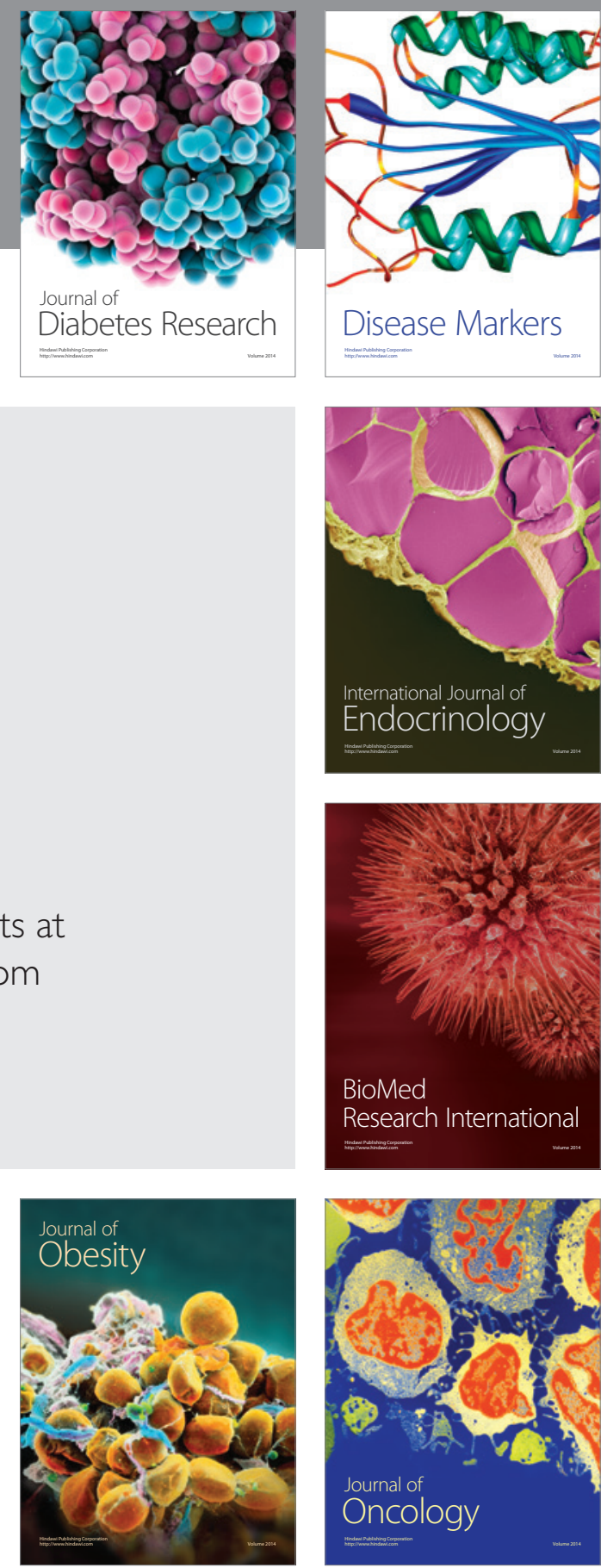

Disease Markers
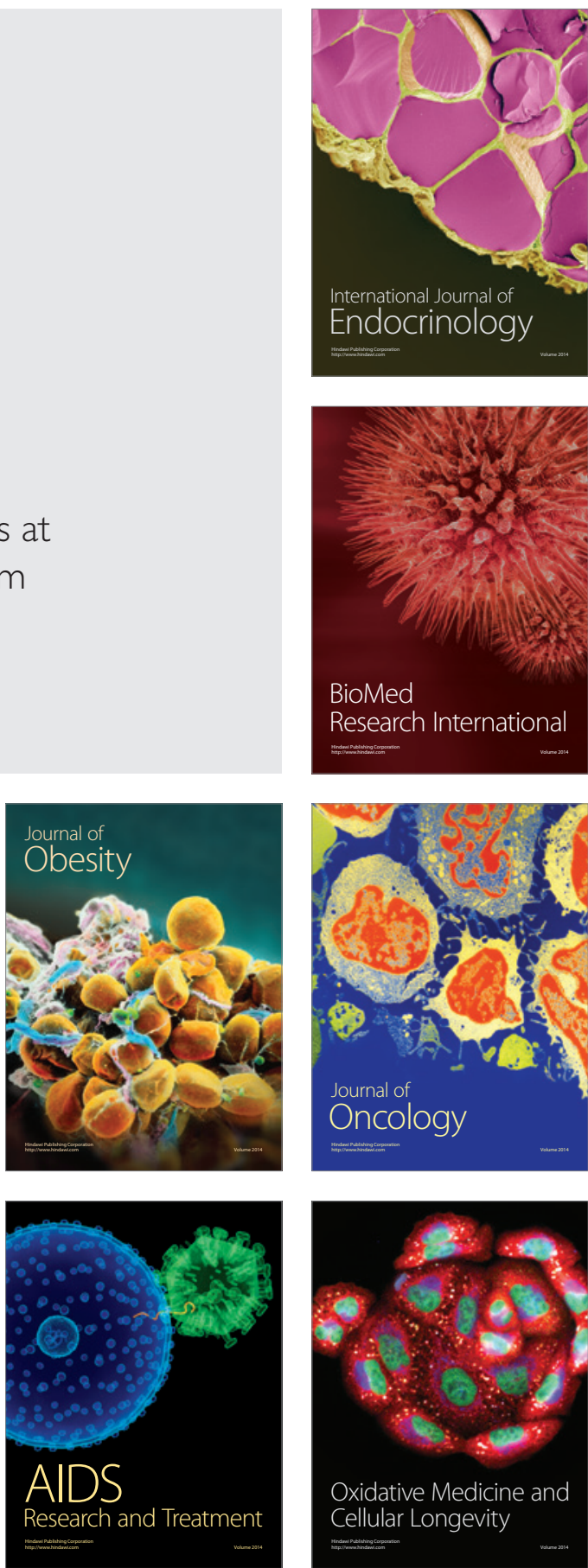